\title{
Association Between Metabolic Associated Fatty Liver Disease and Chronic Kidney Disease: A Cross- Sectional Study from NHANES 2017-2018
}

\author{
Yunlei Deng ${ }^{1, *}$ \\ Qianwen Zhao ${ }^{2, *}$ \\ Rong Gong' \\ 'Department of Nephrology, The \\ Chengdu Second Affiliated Hospital of \\ Chongqing Medical University, The Third \\ People's Hospital of Chengdu, Chengdu, \\ People's Republic of China; ${ }^{2}$ Department \\ of Gastroenterology and Hepatology, \\ West China Hospital, Sichuan University, \\ Chengdu, People's Republic of China \\ *These authors contributed equally to \\ this work
}

Correspondence: Rong Gong Department of Nephrology, The Chengdu Second Affiliated Hospital of Chongqing Medical University, The Third People's Hospital of Chengdu, 37\# Qinglong Street, Qingyang District, Chengdu, Sichuan Province, People's Republic of China

Tel +86I5982I74685

Email gongr2006@sina.com
Purpose: In 2020, an international expert consensus proposed a novel concept, defined as metabolic associated fatty liver disease (MAFLD). We aimed to investigate the association between MAFLD and chronic kidney disease (CKD).

Methods: A total of 4869 subjects with demographic data, laboratory tests, and ultrasound transient elastography from National Health and Nutrition Examination Surveys of the United States (NHANES) 2017-2018 were included in the study. Statistical analysis was performed to test the independent association between the demographic data, laboratory tests, and non-invasive liver fibrosis scores in subjects with different subgroups of MAFLD. Results: A total of 4869 subjects were identified in the NHANES 2017-2018, of which 1032 (21.2\%) subjects were diagnosed with CKD. There was a higher prevalence of CKD in MAFLD subjects than in non-MALFD subjects $(22.2 \%$ vs $19.1, \mathrm{p}=0.048)$. After $1: 1$ propensity score matching by gender, age and race, we enrolled 1983 subjects with MAFLD diagnosed based on liver ultrasound transient elastography and 1983 PS-matched subjects without MAFLD. MAFLD was not independently associated with CKD after PSM. Further investigation showed that age (OR: 1.05, 95\% CI: 1.03 1.05, p<0.001), hypertension (OR: 1.66, 95\% CI: 1.38 2.00, $\mathrm{p}<0.001$ ), DM (OR: 2.21, 95\% CI: 1.89 3.11, p<0.001), hyperuricemia (OR: $1.91,95 \% \mathrm{CI}$ : 1.55 2.36, $\mathrm{p}<0.001$ ), ALP (OR: 1.00, 95\% CI: 1.00 1.01, $\mathrm{p}=0.010$ ), and FIB-4 score (OR: 1.23, 95\% CI: $1.05 \sim 1.01, \mathrm{p}=0.011$ ) were independently associated with $\mathrm{CKD}$. In the subgroup analysis, the subgroups of MAFLD complicated with DM, age, hypertension, and hyperuricemia were independently related to the incidence of CKD. In the subgroup of DM without MAFLD, age, hyperuricemia, ALP, and NFS score were independently related to the incidence of CKD. In the subgroup of MAFLD without DM, age, hypertension, hyperuricemia, and ALP were independently related to the incidence of CKD.

Conclusion: Based on the NHANES 2017-2018, MAFLD was not independently associated with CKD. Thus, the link between MAFLD and CKD may be mediated by metabolic abnormalities, such as diabetes mellitus and hyperuricemia.

Keywords: metabolic associated fatty liver disease, chronic kidney disease, diabetes mellitus, hyperuricemia

\section{Introduction}

Non-alcoholic fatty liver disease (NAFLD) is one of the most common chronic liver diseases, posing a significant health and economic burden. ${ }^{1,2}$ However, due to the increasing prevalence of metabolic dysfunction, the diagnosis of NAFLD might be outof-date. Hence, an international expert consensus in 2020 proposed a novel concept, defined as Metabolic Associated Fatty Liver Disease (MAFLD). ${ }^{3,4}$ The diagnostic 
criteria of MAFLD are significantly distinguished from NAFLD. The main difference of diagnostic criteria between MAFLD and NAFLD is that MAFLD diagnosis does not exclude patients with secondary etiologies of hepatic steatosis in the former disease, such as alcohol consumption, hepatitis virus infection, autoimmune liver diseases, etc. Due to which the prevalence of MAFLD can be higher than that of NAFLD with a prevalence of about $25-30 \%$. ${ }^{1,5}$

Chronic kidney disease (CKD), which is another worldwide public health problem affecting $10-15 \%$ of the whole population, is a progressive chronic condition which manifests with either abnormalities of renal structure or function for more than 3 months. ${ }^{6,7}$ Both NAFLD and $\mathrm{CKD}$ have become major public health problems owing to their increasing prevalence and incidence. Since patients with NAFLD exhibit risk factors for CKD, such as visceral obesity, arterial hypertension, and type 2 diabetes mellitus (T2DM), NAFLD and CKD might be closely connected. However, in previous cross-sectional studies and cohort studies, the relationship between NAFLD and CKD was controversial. ${ }^{8-13}$ Among them, two metaanalyses and systematic reviews supported the assertion that NAFLD increases risk of CKD. ${ }^{14,15}$

Recently, a study compared the characteristics between MAFLD and NAFLD based on National Health and Nutrition Examination Surveys (NHANES) 1988-1994. The study found that MAFLD patients had higher rate of metabolic comorbidities and more severe liver injury. ${ }^{16}$ Besides, a recent study conducted by Sun et al investigated the relationship between MAFLD and CKD based on NHANES 1988-1994, and revealed that MAFLD could identify CKD better than NAFLD. ${ }^{17}$ Hence, MAFLD could give physicians more chance to intervene in the disease earlier. However, this study by Sun et al was conducted with out-of-date data. Herein, we conducted a cross-sectional study based on latest NHANES data, the NHANES 2017-2018, to explore the relationship between MAFLD and CKD, thus reflecting real-world data more accurately.

\section{Methods}

\section{Study Population}

The study population was extracted from the latest National Health and Nutrition Examination Surveys 2017-2018 (NHANES 2017-2018), conducted by the National Center for Health Statistics of the Centers for Disease Control and Prevention of the United States.
Although the NHANES 1988-1994 is the most studied database of NAFLD, the data are outdated - more than 20 years, a period during which the disease fingerprint has changed greatly. The National Center for Health Statistics Research Ethics Review Board approved the NHANES protocol and informed consent was obtained from all subjects. The datasets and further information on NHANES are available at https://wwwn.cdc.gov/nchs/nhanes/.

\section{Diagnostic Criteria}

Based on the international expert consensus statement, diagnosis of MAFLD is defined by liver steatosis diagnosed by ultrasound transient elastography and one of the following conditions, namely overweight or obesity, type 2 diabetes mellitus and lean/normal weight complicated with no less than two metabolic risk abnormalities. Overweight or obesity is defined as body mass index (BMI) $\geq 25 \mathrm{~kg} / \mathrm{m}^{2}$ in Caucasians or BMI $\geq 23 \mathrm{~kg} / \mathrm{m}^{2}$ in Asians. Type 2 diabetes mellitus is defined according to widely accepted international criteria. Metabolic risk abnormalities are listed below, 1) waist circumference $\geq 102 / 88 \mathrm{~cm}$ in Caucasian men and women (or $\geq 90$ / $80 \mathrm{~cm}$ in Asian men and women); 2) blood pressure $\geq 130 / 85 \mathrm{mmHg}$ or specific drug treatment; 3) plasma triglycerides $\geq 150 \mathrm{mg} / \mathrm{dl}$ or specific drug treatment; 4) plasma HDL-cholesterol $<40 \mathrm{mg} / \mathrm{dl}$ for men and $<50 \mathrm{mg}$ / dl for women or specific drug treatment; 5) prediabetes (ie, fasting glucose levels 100 to $125 \mathrm{mg} / \mathrm{dl}$, or 2-hour post-load glucose levels 140 to $199 \mathrm{mg} / \mathrm{dl}$ or HbA1c $5.7 \%$ to $6.4 \%$; 6) homeostasis model assessment of insulin resistance score $\geq 2.5$; 7) plasma high-sensitivity C-reactive protein level $>2 \mathrm{mg} / \mathrm{L}^{3}$.

In the NHANES database, steatosis was assessed by liver ultrasound transient elastography, which was performed by ultrasound and the vibration controlled transient elastography. According to description by the NHANES 2017-2018 data documentation, participants were excluded if they 1) were unable to lie down on the exam table, 2) were pregnant (or unsure if pregnant) at the time of their exam, or urine could not be obtained to test for pregnancy, 3) had an implanted electronic medical device, or 4) were wearing a bandage or had lesions on the right side of their abdomen by the ribs (where measurements would be taken). We defined hepatic steatosis as median FibroScan CAP $\geq 240 \mathrm{~dB} / \mathrm{m}$.

The evaluation of liver fibrosis score was calculated by fibrosis 4 (FIB-4) score and NAFLD fibrosis score (NFS). Their formulas are as follows, FIB- $4=$ age (years) $\times$ AST 
$(\mathrm{IU} / \mathrm{L}) \quad /\left(\mathrm{PLT}\left(10^{\wedge} 9 / \mathrm{L}\right) \times \sqrt{ }(\mathrm{ALT}(\mathrm{IU} / \mathrm{L}))\right), \quad \mathrm{NFS}=-1.675+$ $0.037 \times$ age $($ years $)+0.094 \times \mathrm{BMI}\left(\mathrm{kg} / \mathrm{m}^{2}\right)+1.13 \times$ impaired fasting glucose /diabetes (yes $=1$, no $=0$ ) $+0.99 \times \mathrm{AST} /$ ALT $-0.013 \times$ platelet $\left(\times 10^{9} / 1\right)-0.66 \times$ albumin $(\mathrm{g} / \mathrm{dl})$. The lower cutoff and the upper cutoff, for FIB-4 were 1.3 and 2.67 , for NFS were -1.455 and 0.676 , respectively. We defined the severity of fibrosis as grade 1 , grade 2 and grade 3 , according to the cutoff value.

The definition of CKD is either an estimated glomerular filtration rate (eGFR) of $\leq 60 \mathrm{~mL} / \mathrm{min} / 1.73 \mathrm{~m}^{2}$ or the presence of albuminuria. The calculation of eGFR is based on Chronic Kidney Disease Epidemiology Collaboration (CKD-EPI) equation (2009) for standardized creatinine. The equation is $\quad e G F R=141 * \min \left(\frac{S_{C r}}{\kappa}, 1\right)^{\alpha} *$ $\max \left(\frac{S_{C r}}{\kappa}, 1\right)^{-1.209} * 0.993^{\text {Age }} * 1.108[$ if female $] * 1.159$ $\left(\mathrm{S}_{\mathrm{Cr}}(\right.$ Serum creatinine $\left.)\right)=\mathrm{mg} / \mathrm{dL}, \kappa=0.7$ (females) or 0.9 (males), $\alpha=-0.329$ (females) or -0.411 (males), $\mathrm{min}=$ indicates the minimum of $\mathrm{S}_{\mathrm{Cr}} / \kappa$ or $1, \max =$ indicates the maximum of $\mathrm{S}_{\mathrm{Cr}} / \kappa$ or 1 , age $=$ years). ${ }^{18}$ Albuminuria was defined as urinary albumin-to-creatinine ratio (UACR) $\geq 30 \mathrm{mg} / \mathrm{g}$. As described by NHANES 2017-2018, urinary albumin was measured by solid-phase fluorescent immunoassay, urinary creatinine was measured by enzymatic method.

\section{Other Diagnostic Criteria}

Hypertension was defined by physician diagnosis of hypertension or the use of antihypertensive medications or mean artery systolic pressure $\geq 140 \mathrm{mmHg}$ or diastolic pressure $\geq 90 \mathrm{mmHg}$. Diabetes mellitus was defined as a physician diagnosis of diabetes or the use of antidiabetic medications. The definition of hyperuricemia is based on female $\geq 360 \mu \mathrm{mol} / \mathrm{L}$, and male $\geq 420 \mu \mathrm{mol} / \mathrm{L}$.

\section{Variables}

From the dataset, we obtained the following data, 1) demographic data: age in years at time of screening, gender, race, pregnancy status, etc. 2) Body measurement data: weight, standing height, waist circumference, hip circumference, etc. 3) Standard blood biochemistry profile data: alanine aminotransferase (ALT), albumin, globulin, alkaline phosphatase (ALP), creatinine, calcium, potassium, osmolality, etc. Besides, we had collected data about various questionnaire data, prescription data, laboratory data, and examination data.

\section{Statistical Analysis}

We obtained the data from the website mentioned previously. Then we merged the data by the unique respondent sequence number, using RStudio (Version 1.3.959). The included subjects were divided into two groups: the MAFLD group and non-MAFLD group. To balance the confounding factors, we calculated propensity score matching (PSM) using logistic regression model for the following variables: gender, age, and race. The two groups were matched 1:1 with a caliper of 0.02 .

The continuous variables were compared using Student's $t$-test, and categorical variables using $\chi^{2}$ test. Binary regression analysis was used to assess independent risk factors. All tests were two-tailed and results with a $\mathrm{p}<$ 0.05 were considered statistically significant. All calculations were conducted by SPSS software (version 24.0, IBM Corp., Armonk, NY, USA).

\section{Results}

\section{Baseline Characteristics of Subjects}

9254 subjects in total and 1526 (16.5\%) CKD subjects from the NHANES 2017-2018 dataset were included. Among them, we excluded subjects who were aged less than 20 years old, pregnant, lacked creatinine and liver ultrasound transient elastography (Figure 1). A final 4869 subjects, of which 1032 (21.2\%) subjects had been diagnosed with CKD, were included.

\section{Demographic Characteristics' Comparison Between Subjects with or without MAFLD}

The comparison between subjects before and after propensity score matching (PSM) is displayed in Table 1. Before PSM, the number of MAFLD subjects with CKD was 614 (22.2\%), which was higher than that of non-MAFLD subjects $(22.2 \%$ vs 19.1, $\mathrm{p}=0.048$ ). However, after PSM, the two groups showed similar prevalence of CKD (MAFLD: $20.6 \%$ vs nonMAFLD: $20.5 \%, p=0.969)$. Age, gender, and race showed significant difference between subjects with or without MAFLD before PSM, as shown in Table 1, and these indexes became comparable after PSM. Concerning the diagnostic criteria of MAFLD, before PSM participants with MAFLD tended to be overweight $(95.1 \%$ vs $52.2 \%$, $\mathrm{p}<0.001)$, have hypertension $(36.1 \%$ vs $25.1 \%, \mathrm{p}<0.001)$ and diabetes mellitus $(24.1 \%$ vs $9.4 \%, \mathrm{p}<0.001)$. After PSM, compared with non-MAFLD subjects, MAFLD subjects had a higher prevalence of being overweight (95.4\% vs $52.7 \%, \mathrm{p}<0.001)$, having hypertension $(34.2 \%$ vs $26.5 \%$, $\mathrm{p}<0.001)$ and DM (20.3\% vs 9.9\%, $<<0.001)$. 


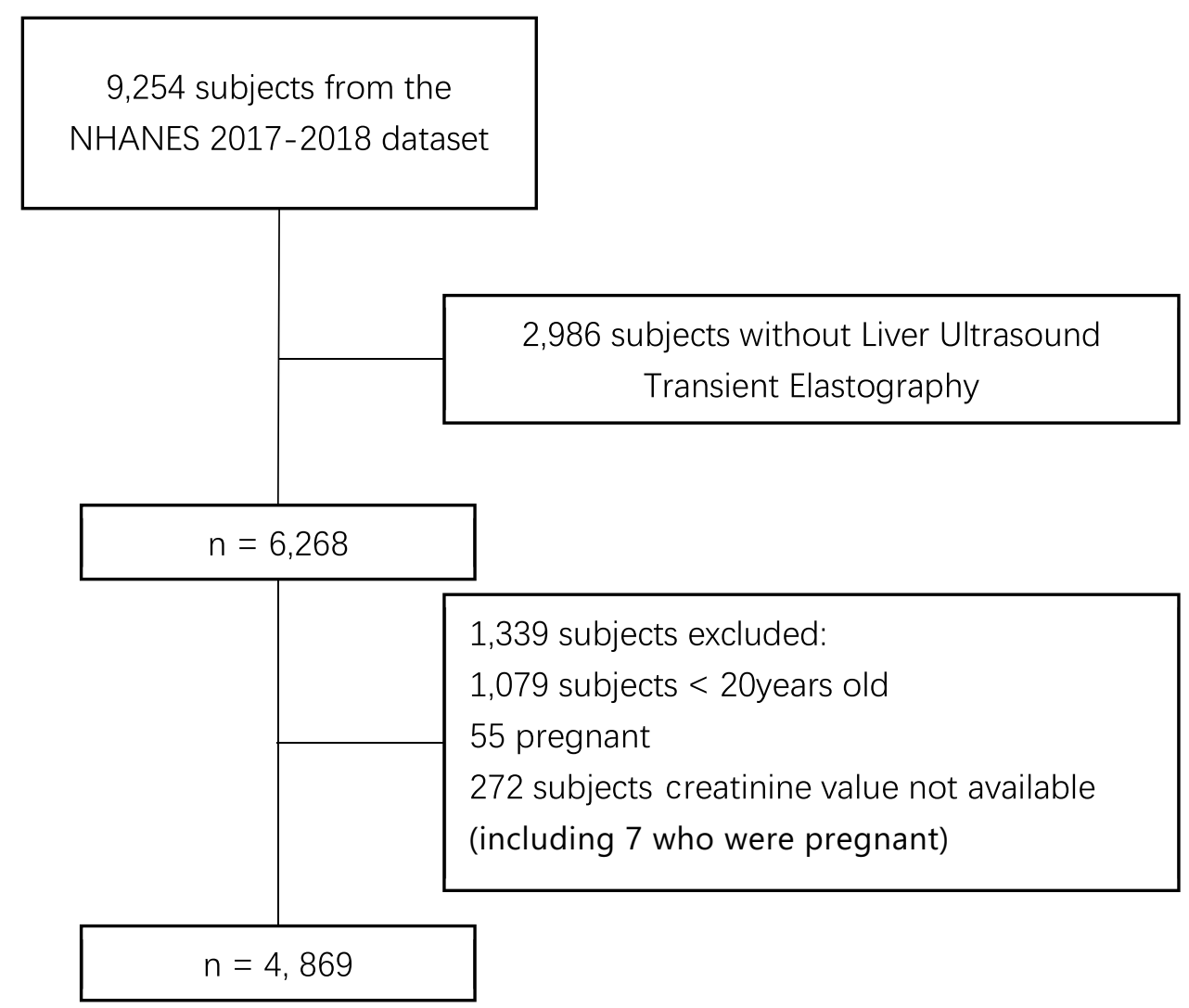

Figure I Flowchart of the included 4869 subjects from 9254 subjects in the NHANES 2017-2018 dataset. Abbreviation: NHANES, National Health and Nutrition Examination Surveys.

\section{Laboratory Data Comparison Between Subjects with or without MAFLD}

We collected subjects' laboratory data, including hsCRP, creatinine, ALT, GGT, ALP, HbA1c, HbA1c, total cholesterol, TG, LDL-C, uric acid, and urine albumin creatinine ratio. According to the diagnostic criteria mentioned previously, we presumed subjects with abnormal values could be diagnosed with hyperuricemia, hypercholesterolemia, hypertriglyceridemia, low HDL level or albuminuria as shown in Table 1. Before PSM, compared with non-MAFLD subjects, MAFLD subjects showed higher prevalence ratio in $\mathrm{CKD}$, albuminuria, hyperuricemia, hypertriglyceridemia, low HDL-C level, GGT, ALT, FIB-4 score $\geq 1.3$, NFS score $\geq-1.455$, and higher value in hsCRP, ALT, ALP, GGT, FIB-4 score, and NFS score. After PSM, there were no significant differences of eGFR, gender, race, hsCRP, ALT, and GGT levels between two groups.

\section{Associated Factors of CKD in the Univariate and Multivariate Binary Regression Analysis After PSM}

To investigate the independently associated factors of CKD subjects, we performed univariate and multivariate binary regression analysis in the subjects after PSM, as shown in Table 2. Binary regression analysis was adjusted for age, smoking history, overweight, hypertension, DM, hyperuricemia, hyperuricemia, hypertriglyceridemia, hsCRP, ALT, ALP, FIB-4 score, and NFS score. The results showed that age (OR: 1.05, 95\% CI: 1.03 1.05, p<0.001), hypertension (OR: 1.66, 95\% CI: 1.38 2.00, p<0.001), DM (OR: $2.21,95 \%$ CI: 1.89 3.11, $\mathrm{p}<0.001$ ), hyperuricemia (OR: $1.91,95 \% \mathrm{CI}$ : 1.55 2.36, $\mathrm{p}<0.001$ ), ALP (OR: 1.00, 95\% CI: 1.00 1.01, $\mathrm{p}=0.010$ ), and FIB-4 score (OR: 1.23, 95\% CI: 1.05 1.01, $\mathrm{p}=0.011$ ) were independently associated with CKD.

We further investigated the composition of different fibrosis scores of CKD and non-CKD subjects (Figure 2). The percentages of grade 1 fibrosis evaluated by FIB-4 or NFS, in non-CKD group were $76 \%$ and $60 \%$, but in CKD group the percentages were $45 \%$ and $25 \%$, respectively. The percentages of grade 2 fibrosis were $22 \%$ and $34 \%$ in non-CKD group, $45 \%$ and $52 \%$ in CKD group. For grade 3 fibrosis, the percentages were $2 \%$ and $6 \%$ in non-CKD group, $10 \%$ and $24 \%$ in CKD group. Thus, compared with non-CKD subjects, CKD subjects had higher grade of fibrosis, which is more obvious in grade 3 fibrosis. 
Table I Baseline Characteristics Before and After Propensity Score (PS) Matching

\begin{tabular}{|c|c|c|c|c|c|c|}
\hline & \multicolumn{3}{|c|}{ Before PSM } & \multicolumn{3}{|c|}{ After PSM } \\
\hline & $\begin{array}{l}\text { Non-MAFLD } \\
(n=2104)\end{array}$ & $\begin{array}{l}\text { MAFLD } \\
(n=2765)\end{array}$ & $\mathbf{p}$ & $\begin{array}{l}\text { Non-MAFLD } \\
(n=1983)\end{array}$ & $\begin{array}{l}\text { MAFLD } \\
(n=1983)\end{array}$ & $\mathbf{p}$ \\
\hline CKD n (\%) & 418 (19.9) & $614(22.2)$ & 0.048 & $408(20.6)$ & $407(20.5)$ & 0.969 \\
\hline Albuminuria n (\%) & 242 (II.5) & $427(15.4)$ & $<0.001$ & $233(11.7)$ & $289(14.3)$ & $0.016^{*}$ \\
\hline eGFR & I07.7 (34.3) & $76.8(47.4)$ & $<0.001$ & $100.1(40.4)$ & $103.0(40.4)$ & 0.085 \\
\hline Age, years $\bar{x} \pm s$ & $49.5(19.1)$ & $53.4(16.2)$ & $<0.001$ & $50.8(18.8)$ & $5 I .1(16.0)$ & 0.636 \\
\hline Male n (\%) & $923(43.9)$ & $1442(52.2)$ & $<0.001$ & $1068(46.1)$ & $1028(51.8)$ & 0.203 \\
\hline Race n (\%) & & & $<0.001$ & & & 0.558 \\
\hline Mexican American n (\%) & $209(9.9)$ & $456(16.5)$ & & $209(10.5)$ & $24 \mid(12.2)$ & \\
\hline Other Hispanic n (\%) & $176(8.4)$ & $289(10.5)$ & & $176(8.9)$ & $175(8.8)$ & \\
\hline Non-Hispanic White n (\%) & $769(36.5)$ & 938 (33.9) & & $718(36.2)$ & $692(34.9)$ & \\
\hline Non-Hispanic Black n (\%) & $542(25.8)$ & $559(20.2)$ & & $477(24.1)$ & $484(24.4)$ & \\
\hline Non-Hispanic Asian n (\%) & $296(14.1)$ & $384(13.9)$ & & $295(14.9)$ & $274(13.8)$ & \\
\hline Other Race n (\%) & $112(5.3)$ & $139(5.0)$ & & $108(5.4)$ & $117(5.9)$ & \\
\hline Overweight n (\%) & $1059(52.2)$ & $2630(95.1)$ & $<0.001$ & $1004(52.7)$ & $1892(95.4)$ & $<0.001 *$ \\
\hline Smoking n (\%) & $870(4 I .3)$ & $1|8|(42.7)$ & 0.340 & $823(42.0)$ & $834(42.1)$ & 0.949 \\
\hline Hypertension n (\%) & $529(25.1)$ & $998(36.1)$ & $<0.001$ & $524(26.5)$ & $679(34.2)$ & $<0.001 *$ \\
\hline DM n (\%) & $198(9.4)$ & $593(24.1)$ & $<0.001$ & $196(9.9)$ & $403(20.3)$ & $<0.001 *$ \\
\hline Hyperuricemia n (\%) & $291(13.8)$ & $686(24.8)$ & $<0.001$ & $280(14.1)$ & $496(25.0)$ & $<0.001 *$ \\
\hline Hypertriglyceridemia n (\%) & $383(18.2)$ & I24I (44.9) & $<0.001$ & $382(19.3)$ & $859(43.3)$ & $<0.001 *$ \\
\hline Low HDL-C n (\%) & $223(10.6)$ & $15(0.5)$ & $<0.001$ & $197(9.9)$ & II (0.6) & $<0.001 *$ \\
\hline Creatinine, $\mu \mathrm{mol} / \mathrm{L} \bar{x} \pm s$ & $80.3(38.7)$ & $80.6(44.9)$ & 0.805 & 8I.I (39.6) & $79.7(47.3)$ & 0.315 \\
\hline hsCRP, $\mathrm{mg} / \mathrm{L} \bar{x} \pm s$ & $3.4(8.1)$ & $4.8(8.2)$ & $<0.001$ & $3.4(8.3)$ & $5.1(8.3)$ & $<0.001 *$ \\
\hline $\mathrm{ALT}$ IU/L $\bar{x} \pm s$ & $18.8(14)$ & $25.0(17.8)$ & $<0.001$ & $21.6(13.8)$ & $22.3(11.9)$ & 0.085 \\
\hline ALP IU/L $\bar{x} \pm s$ & $77(29.9)$ & $81.7(25.2)$ & 0.005 & $77.6(30.1)$ & $82.0(25.3)$ & $<0.001 *$ \\
\hline GGT IU/L $\bar{x} \pm s$ & $28.5(50.1)$ & $35.9(42.6)$ & $<0.001$ & $159.3(35.4)$ & $160.4(34.8)$ & 0.304 \\
\hline FIB-4 Score $\bar{x} \pm s$ & $1.18(0.88)$ & I.0I (I.0I) & 0.215 & $\mathrm{I} .06(0.7 \mathrm{I})$ & $1.2(0.88)$ & $<0.001 *$ \\
\hline FIB-4 Category & & & 0.009 & & & $<0.001 *$ \\
\hline FIB-4 $<$ I. 3 & $1402(66.8)$ & $1914(69.5)$ & & I $463(74.0)$ & $1284(64.9)$ & \\
\hline I. $3 \leq$ FIB- $4<2.67$ & $596(28.4)$ & $75 I$ (27.2) & & $495(23.2)$ & $595(30.1)$ & \\
\hline FIB-4 $\geq 2.67$ & $100(4.8)$ & $88(3.2)$ & & $55(2.8)$ & $99(5.0)$ & \\
\hline NFS Score $\bar{x} \pm s$ & $-1.79(1.54)$ & $-1.01(1.55)$ & $<0.001$ & $-1.7(1.53)$ & $-1.2(1.57)$ & $<0.001 *$ \\
\hline NFS Category & & & $<0.001$ & & & $<0.001 *$ \\
\hline NFS $<-1.455$ & $1268(62.7)$ & II 65 (42.3) & & II 58 (60.9) & $903(45.7)$ & \\
\hline$-1.455 \leq \mathrm{NFS}<0.676$ & $617(30.5)$ & $1239(45.0)$ & & $607(31.9)$ & $839(42.2)$ & \\
\hline NFS $\geq 0.676$ & $136(6.5)$ & $349(12.7)$ & & $136(7.2)$ & 235 (11.9) & \\
\hline
\end{tabular}

Notes: *A significant difference between groups. Categorical values are shown as $\mathrm{n}(\%)$. Continuous variables are shown as mean \pm standard deviation.

Abbreviations: ALP, alkaline phosphatase; ALT, alanine aminotransferase; AST, aspartate aminotransferase; BMI, body mass index; CKD, chronic kidney disease; DM, diabetes mellitus; eGFR, estimated glomerular filtration rate; FIB-4, fibrosis-4; GGT, $\gamma$-glutamyl transpeptidase; HDL-C, high-density lipoprotein cholesterol; hsCRP, highsensitivity C-reactive protein; NFS, NAFLD fibrosis score. 
Table 2 Univariate and Logistic Regression Analysis in Patients Based on the PSM

\begin{tabular}{|c|c|c|c|c|c|}
\hline & Non-CKD $(n=3 \mid 5 I)$ & CKD $(n=815)$ & $\mathrm{p}$ value & Odds Ratio (95\% Cl) & p value \\
\hline MAFLD & $1576(50.0)$ & 407 (49.9) & 0.969 & & \\
\hline Age $\bar{x} \pm s$ & $47.9(16.5)$ & $63.6(15.3)$ & $<0.001$ & $1.04(1.03 \sim 1.05)$ & $<0.00 I^{*}$ \\
\hline Male n (\%) & $1668(52.9)$ & $428(52.5)$ & 0.844 & & \\
\hline Overweight n (\%) & $2282(73.5)$ & $614(78.4)$ & 0.004 & $0.98(0.77 \sim 1.25)$ & 0.723 \\
\hline Smoking n (\%) & $1272(40.4)$ & $394(48.3)$ & $<0.001$ & $0.92(0.77 \sim 1.10)$ & 0.388 \\
\hline Hypertension n (\%) & $778(24.7)$ & $425(52.1)$ & $<0.001$ & $1.66(1.38 \sim 2.00)$ & $<0.00 I^{*}$ \\
\hline DM n (\%) & $329(10.4)$ & $270(33.1)$ & $<0.001$ & $2.21(1.89 \sim 3.11)$ & $<0.00 I^{*}$ \\
\hline Hyperuricemia n (\%) & $484(15.4)$ & $292(35.8)$ & $<0.001$ & 1.91 (1.55 2.36) & $<0.00 I^{*}$ \\
\hline Hypertriglyceridemia n (\%) & $939(29.8)$ & $302(37.1)$ & $<0.001$ & $0.83(0.69 \sim 1.00)$ & 0.055 \\
\hline Low HDL n (\%) & $159(5.0)$ & $49(6.0)$ & 0.270 & & \\
\hline High hsCRP n (\%) & 8I.I (39.6) & $79.7(47.3)$ & 0.315 & & \\
\hline ALT IU/L $\bar{x} \pm s$ & $22.2(15.9)$ & $20.8(17.0)$ & 0.028 & $1.00(1.00 \sim 1.00)$ & 0.231 \\
\hline ALP IU/L $\bar{x} \pm s$ & $78.2(27.0)$ & $85.8(30.2)$ & $<0.001$ & $1.00(1.00 \sim 1.01)$ & $0.010^{*}$ \\
\hline FIB-4 Score & $1.0(0.70)$ & $1.6(1.0)$ & $<0.001$ & $1.23(1.05 \sim 1.45)$ & 0.011 \\
\hline $\begin{array}{l}\text { FIB-4 Category } \\
\qquad \begin{array}{l}\text { FIB-4 }<\text { I. } 3 \\
\text { I. } 3 \leq \text { FIB-4 }<2.67 \\
\text { FIB-4 } \geq 2.67\end{array}\end{array}$ & $\begin{array}{l}2382(75.8) \\
684(21.8) \\
75(2.4)\end{array}$ & $\begin{array}{l}365(44.8) \\
370(45.5) \\
79(9.7)\end{array}$ & $<0.001$ & & \\
\hline NFS score & $-1.7(1.4)$ & $-0.40(1.6)$ & $<0.001$ & $1.09(0.98 \sim 1.20)$ & 0.102 \\
\hline $\begin{array}{l}\text { NFS Category } \\
\qquad \begin{array}{l}\text { NFS }<-1.455 \\
-1.455 \leq \text { NFS }<0.676 \\
\text { NFS } \geq 0.676\end{array}\end{array}$ & $\begin{array}{l}\mid 870(60.7) \\
|04|(33.6) \\
\mid 85(6.0)\end{array}$ & $\begin{array}{l}194(24.4) \\
405(51.8) \\
186(23.8)\end{array}$ & $<0.001$ & & \\
\hline
\end{tabular}

Notes: *A significant difference between groups. Categorical values are shown as $\mathrm{n}(\%)$. Continuous variables are shown as mean \pm standard deviation.

Abbreviations: ALP, alkaline phosphatase; ALT, alanine aminotransferase; AST, aspartate aminotransferase; BMI, body mass index; CKD, chronic kidney disease; DM, diabetes mellitus; eGFR, estimated glomerular filtration rate; FIB-4, fibrosis-4; GGT, $\gamma$-glutamyl transpeptidase; HDL-C, high-density lipoprotein cholesterol; hsCRP, highsensitivity C-reactive protein; NFS, NAFLD fibrosis score; PSM, propensity score matching.

\section{Associated Factors of CKD in MAFLD Subjects Complicated with DM}

Associations Between CKD in Subjects with MAFLD Complicated with DM

In subjects with MAFLD complicated with DM, age (OR: $1.06,95 \%$ CI: 1.02 1.09, $\mathrm{p}<0.001$ ), hypertension (OR: 1.78, 95\% CI: 1.16 2.58, $\mathrm{p}=0.008$ ), and hyperuricemia (OR: 2.08 , $95 \%$ CI: $1.03 \sim 3.32, \mathrm{p}=0.008$ ) were independently associated factors of CKD (Table 3). However, ALP, FIB-4 score, and NFS score were not independently associated with CKD.

Associations Between CKD in DM Subjects without MAFLD In subjects with DM without MAFLD, age (OR: 1.06, 95\% CI: 1.02 1.09, $\mathrm{p}=0.002$ ), hyperuricemia (OR: 3.52,
95\% CI: 1.52 8.15, $\mathrm{p}=0.003$ ), ALP (OR: $1.02,95 \%$ CI: 1.00 1.03, $\mathrm{p}=0.014$ ), and NFS score (OR: $1.51,95 \% \mathrm{CI}$ : $1.01 \sim 2.15, \mathrm{p}=0.022)$ were independently associated factors of CKD (Table 4). However, hypertension and FIB-4 were not independently associated with CKD (Table 4).

Associations Between CKD in MAFLD Subjects without DM In subjects with MAFLD without DM, age (OR: 1.02, 95\% CI: $1.01 \sim 1.04, \mathrm{p}=0.002$ ), hypertension (OR: 2.01, 95\% CI: $1.54 \sim 2.86, \mathrm{p}<0.01$ ), hyperuricemia (OR: $2.25,95 \% \mathrm{CI}$ : 1.30 3.07, $\mathrm{p}<0.001$ ), and ALP (OR: 1.01, 95\% CI: $1.00 \sim 1.01, \mathrm{p}=0.021)$ were independently associated factors of CKD (Table 4). However, FIB-4 score and NFS score were not independently associated with CKD (Table 5). 


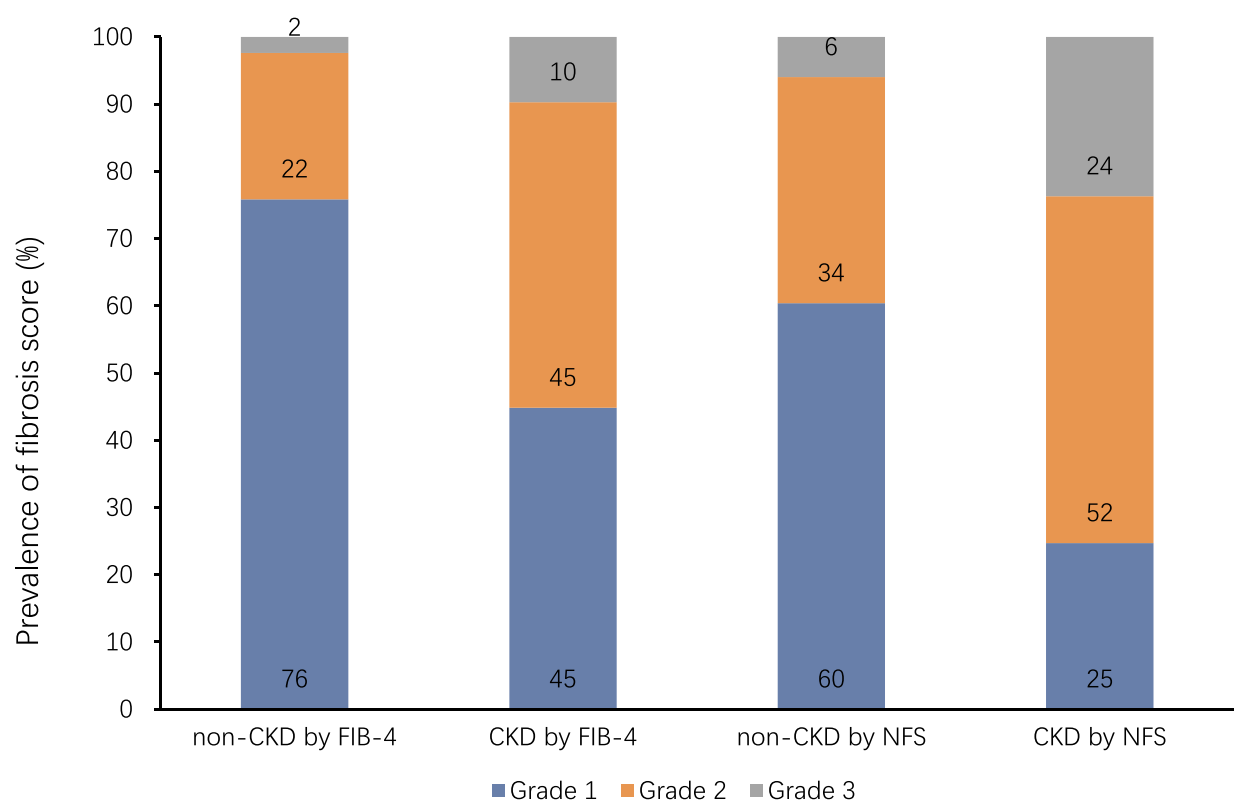

Figure 2 Fibrosis classification based on FIB-4 or NFS scores in CKD and non-CKD subjects. Abbreviations: CKD, chronic kidney disease; FIB-4, fibrosis-4; NFS, NAFLD fibrosis score.

\section{Discussion}

In this cross-sectional study, a total of 4869 subjects and $1032(21.2 \%)$ CKD subjects meeting the inclusion criteria from the NHANES 2017-2018 dataset were included. Our results showed that the MAFLD group had higher prevalence of CKD than non-MAFLD group ( $22.2 \%$ vs $19.1 \%, \mathrm{p}=0.048)$ before PSM. However, after PSM by age, gender, and race, the prevalence of CKD between MAFLD group and non-MAFLD group was similar. The logistic analysis showed that the comorbidities of MAFLD including hypertension and diabetes mellitus were independently associated with CKD, while MAFLD was not independently associated with CKD. Meanwhile, apart from comorbidities of MAFLD, age, hyperuricemia, and FIB-4 score were independently

Table 3 Associations Between CKD in Subjects with MAFLD Complicated with DM

\begin{tabular}{|l|l|l|}
\hline & p-value & Odds Ratio (95\% Cl) \\
\hline Age & $<0.001^{*}$ & $1.04(1.02 \sim 1.07)$ \\
Hypertension & $0.008^{*}$ & $1.78(1.16 \sim 2.58)$ \\
Hyperuricemia & $0.002^{*}$ & $2.08(1.30 \sim 3.32)$ \\
ALP & 0.138 & $1.01(1.00,1.01)$ \\
FIB-4 Score & 0.985 & $1.00(0.77 \sim 1.29)$ \\
NFS Score & 0.357 & $1.10(0.90 \sim 1.36)$ \\
\hline
\end{tabular}

Note: *A significant difference between groups.

Abbreviations: MAFLD, metabolic associated fatty liver disease; ALP, alkaline phosphatase; FIB-4, fibrosis-4; NFS, NAFLD fibrosis score. associated with CKD. Then, we analyzed subjects diagnosed with either or both DM and MAFLD and found that age and hyperuricemia were independent risk factors for

Table 4 Associations Between CKD in DM Subjects Not Complicated with MAFLD

\begin{tabular}{|l|l|l|}
\hline & p-value & Odds Ratio (95\% Cl) \\
\hline Age & $0.002^{*}$ & $1.06(1.02 \sim 1.09)$ \\
Hypertension & 0.310 & $1.45(0.71 \sim 2.95)$ \\
Hyperuricemia & $0.003^{*}$ & $3.52(1.52 \sim 8.15)$ \\
ALP & $0.014^{*}$ & $1.02(1.00 \sim 1.03)$ \\
FIB-4 Score & 0.264 & $0.79(0.52 \sim 1.19)$ \\
NFS Score & 0.022 & $1.51(1.01 \sim 2.15)$ \\
\hline
\end{tabular}

Note: *A significant difference between groups.

Abbreviations: MAFLD, metabolic associated fatty liver disease; ALP, alkaline phosphatase; FIB-4, fibrosis-4; NFS, NAFLD fibrosis score.

Table 5 Associations Between CKD in Subjects with MAFLD Not Complicated with DM

\begin{tabular}{|l|l|l|}
\hline & p-value & Odds Ratio (95\% CI) \\
\hline Age & $0.002^{*}$ & $1.02(1.01 \sim 1.04)$ \\
Hypertension & $<0.001$ & $2.01(1.54 \sim 2.86)$ \\
Hyperuricemia & $<0.00 I^{*}$ & $2.25(1.30 \sim 3.07)$ \\
ALP & $0.02 I^{*}$ & $1.01(1.00,1.01)$ \\
FIB-4 Score & 0.060 & $1.00(0.77 \sim 1.29)$ \\
NFS Score & 0.494 & $1.05(0.91 \sim 1.21)$ \\
\hline
\end{tabular}

Note: *A significant difference between groups.

Abbreviations: MAFLD, metabolic associated fatty liver disease; ALP, alkaline phosphatase; FIB-4, fibrosis-4; NFS, NAFLD fibrosis score. 
all subgroups. Further investigation revealed that hyperuricemia had highest odds ratio in all the three groups, with odds ratio $>2.00$. Especially, in the DM without MAFLD group, the odds ratio of hyperuricemia reached 3.52 .

MAFLD is a novel concept purposed by an authorized international expert consensus statement, ${ }^{3}$ and a real world analysis indicated that MAFLD is more practical for identifying disease progression than NAFLD. ${ }^{16}$ The major advantage of nomenclature of MAFLD is the connection between known metabolic dysfunction factors and liver fibrosis. The emphasis on metabolic dysfunction could facilitate patient-physician communication and shared decision-making, increase disease awareness, lead to more efficient and effective therapy development. ${ }^{19}$ However, in clinical practice, we cannot ignore the etiologies of liver fibrosis of MAFLD, such as alcohol consumption, autoimmune liver disease, which could be critical for treatment.

Numerous studies have investigated the relationship between CKD and NAFLD. They concluded that NAFLD could be a driving factor for the development and progression of CKD. ${ }^{5}$ The underlying mechanisms include type $2 \mathrm{DM}$, metabolic syndrome, intestinal dysbiosis, visceral adipose dysfunction, platelet activation et al. ${ }^{5}$ In this study, we revealed that MAFLD was not independently associated with CKD after correcting for age, sex and race, which was different from the study by Sun et al. ${ }^{17}$ Hence, more prospective multicenter studies based on larger sample size should be conducted in this field.

Diabetes has become the leading etiology of CKD and end-stage kidney disease, especially in the developed countries and some developing countries. $20 \sim 40 \%$ of patients with diabetes are complicated with CKD, which constitutes $20 \sim 30 \%$ of all CKD patients. ${ }^{6,20-22}$ However, in a crosssectional study based on 4637 Japanese individuals, diabetes was not an independent risk factor for CKD among NAFLD and non-NAFLD patients, which may be due to the limitation of sample size. ${ }^{13}$ What is more, another study of NHANES 1988-1994 dataset indicated that diabetes was not associated with CKD in NAFLD populations. The reason might be that diagnosis of NAFLD is mainly dependent on metabolic abnormalities and those abnormalities cannot alleviate the risk of diabetes. ${ }^{12}$ Hence, more epidemiological research and longitudinal studies are still needed to investigate the association between diabetes mellitus in MAFLD and CKD.
Uric acid has been suggested as a risk factor for various diseases, including cardio-cerebrovascular diseases, CKD, hypertension, insulin resistance, etc. ${ }^{23,24}$ In this study, hyperuricemia is another independently associated factor for CKD. Though the relationship between hyperuricemia and CKD was controversial in the past, the positive relationship has been verified in recent studies and recognized in the guidelines and consensus. ${ }^{11,25}$ Studies have shown that hyperuricemia could promote the development of NAFLD, and increase the risk of developing CKD in those NAFLD patients. ${ }^{26,27}$ In our study, odds ratio (for CKD) of hyperuricemia was highest in all the subgroups analysis, and reached 3.52 in DM without MAFLD subjects. In a Korean cohort study for outcomes in patients with chronic kidney disease (KNOW$\mathrm{CKD}$ ), Oh et al found that hyperuricemia was an independent risk factor for $\mathrm{CKD}$, and the hazard ratio reached $3.590,{ }^{25}$ which was similar to our study.

Hypertension is another concerning public health problem, which is an independently associated factor for CKD in all investigated subjects, but not in sub-group of DM subjects without MAFLD. As hypertension is a common disease affecting almost all human systems, and a leading cause of $\mathrm{CKD},{ }^{28-30}$ the risk in the subgroup might have been eliminated by other factors, such as diabetes, hyperuricemia, being overweight.

Aging is a common risk factor for multi-systemic and multi-organ diseases, including CKD, which has become a public health problem, especially in developed countries. In a 2019 survey by the United States Centers for Disease Control and Prevention, it is reported that incidence of CKD in people aged no less than 65 years was $38 \%$, while in people between $45-64$ it was $13 \%$, and between $18-44$ years it was $7 \% .^{31}$

Although triglycerides and HDL cholesterol are diagnostic parameters in the diagnosis of MAFLD, they did not increase the risk of developing CKD in this study. In fact, research on dyslipidemia and progression of CKD mainly focused on children for the development of obesity related nephropathy. ${ }^{32}$ However, this study only enrolled subjects over 20 years old, and might have missed some data on this part.

FIB-4 and NFS are accepted methods for the estimation of advanced fibrosis as recommended in the major guidelines. ${ }^{33-35}$ However, the accuracy of the two scores can be affected by other confounding factors, such as age and BMI. ${ }^{36,37}$ In this study, we found that percentage of grade 2 or 3 liver fibrosis estimated FIB-4 and NFS was 
higher in the CKD subjects, especially grade 3 . The percentage of FIB-4 and NFS estimated grade 3 in CKD group was quintupled and quadrupled compared to the non-CKD group. In the data after PSM, FIB-4 score was independently associated with CKD (OR: 1.23, 95\% CI: $1.05 \sim 1.45, \mathrm{p}=0.011$ ), but the NFS score was not. However, in the subgroup analysis, NFS score was only independently associated with CKD in the DM subjects without MAFLD (OR: 1.51, 95\% CI: 1.01 2.15, p=0.011), and FIB-4 was not independently associated with CKD in all subgroups. The study conducted by Sun et al, based on NHANES III, found that MAFLD with increased advanced fibrosis scores was associated with higher risk of CKD and albuminuria. ${ }^{17}$ The difference between our study and the study by Sun et al might be the different datasets, which needs further investigation.

The limitations of this study are obvious, including the following: firstly, it is a cross-sectional study which means we lack follow-up, hence the 3 months' renal abnormalities required for the diagnosis of CKD were not available. But the results were consistent with $\mathrm{CKD}$ prevalence. Meanwhile, NHANES is an important epidemiological investigation database. Hence, the results were still reliable and could be justified. Secondly, due to the limitation of NHANES 2017-2018 datasets, parts of diagnostic parameters of MAFLD involving metabolic risk abnormalities are lacking, such as insulin resistance score, 2-hour postload glucose level and types of DM. This might be the flaws of the datasets, but the datasets provide large sample size which could compensate for those drawbacks. Thirdly, the diagnosis of hepatic steatosis in adults was based on liver ultrasound transient elastography, which has been proven to have high specificity and sensitivity, but liver biopsy is the gold standard. Lastly, the risk factors for CKD could be numerous, such as social status, alcohol drinking, insomnia, dementia, but due to the limitation of the dataset we have not used those factors in this study.

\section{Conclusion}

Although MAFLD was not independently associated with CKD in our study, age, hypertension, DM, hyperuricemia, ALP, and FIB-4 score were independently associated with CKD. In subgroups analysis of subjects diagnosed with either or both DM and MAFLD, age and hyperuricemia were independently associated factors for CKD in all subgroups. These results indicate that in clinical practice, we should scrutinize the risk factors of CKD such as hyperuricemia and diabetes for patients diagnosed with
MAFLD, especially in the elderly population. Future large prospective studies should focus on this field to validate the relationship between MAFLD and CKD.

\section{Abbreviations}

NHANES, National Health and Nutrition Examination Surveys; MAFLD, Metabolic Associated Fatty Liver Disease; NAFLD, Nonalcoholic Fatty Liver Disease; ALT, Alanine Aminotransferase; AST, Aspartate Aminotransferase; hsCRP, high sensitivity C-Reactive Protein; UACR, Urine Albumin Creatinine Ratio; BMI, Body Mass Index; GGT, $\gamma$-Glutamyl Transpeptidase; ALP, Alkaline Phosphatase; HDL-C, High-Density Lipoprotein Cholesterol; LDL-C, Low-Density Lipoprotein Cholesterol; TG, Triglycerides; TC, Total Cholesterol; BUN, Blood Urea Nitrogen; HbA1c, Hemoglobin A1c; FPG, Fasting Plasma Glucose; FIB-4, Fibrosis 4; NFS, NAFLD Fibrosis Score.

\section{Author Contributions}

All authors made a significant contribution to the work reported, whether that is in the conception, study design, execution, acquisition of data, analysis and interpretation, or in all these areas; took part in drafting, revising or critically reviewing the article; gave final approval of the version to be published; have agreed on the journal to which the article has been submitted; and agree to be accountable for all aspects of the work.

\section{Funding}

Sichuan Province Medical Association Scientific Research Fund (2019HR33).

\section{Disclosure}

Qianwen Zhao and Yunlei Deng are co-first authors. The authors report no conflicts of interest in this work.

\section{References}

1. Frey S, Patouraux S, Debs T, Gugenheim J, Anty R, Iannelli A. Prevalence of NASH/NAFLD in people with obesity who are currently classified as metabolically healthy. Surg Obes Relat Dis. 2020;16 (12):2050-2057. doi:10.1016/j.soard.2020.07.009

2. Lonardo A, Mantovani A, Lugari S, Targher G. NAFLD in some common endocrine diseases: prevalence, pathophysiology, and principles of diagnosis and management. Int J Mol Sci. 2019;20(11):11. doi:10.3390/ijms20112841

3. Eslam M, Newsome PN, Sarin SK, et al. A new definition for metabolic dysfunction-associated fatty liver disease: an international expert consensus statement. J Hepatol. 2020;73(1):202-209. doi:10.1016/j. jhep.2020.03.039 
4. Eslam M, Sanyal AJ, George J; International Consensus P. MAFLD: a consensus-driven proposed nomenclature for metabolic associated fatty liver disease. Gastroenterology. 2020;158(7):1999-2014 e1991. doi:10.1053/j.gastro.2019.11.312

5. Byrne CD, Targher G. NAFLD as a driver of chronic kidney disease. J Hepatol. 2020;72(4):785-801. doi:10.1016/j.jhep.2020.01.013

6. Zelnick LR, Weiss NS, Kestenbaum BR, et al. Diabetes and CKD in the United States population, 2009-2014. Clin J Am Soc Nephrol. 2017;12(12):1984-1990. doi:10.2215/CJN.03700417

7. Bowe B, Xie Y, Li T, et al. Changes in the US burden of chronic kidney disease from 2002 to 2016: an analysis of the Global Burden of Disease Study. JAMA Netw Open. 2018;1(7):e184412. doi:10. 1001/jamanetworkopen.2018.4412

8. Targher G, Bertolini L, Rodella S, Lippi G, Zoppini G, Chonchol M. Relationship between kidney function and liver histology in subjects with nonalcoholic steatohepatitis. Clin J Am Soc Nephrol. 2010;5 (12):2166-2171. doi:10.2215/CJN.05050610

9. Choe AR, Ryu D-R, Kim HY, et al. Noninvasive indices for predicting nonalcoholic fatty liver disease in patients with chronic kidney disease. BMC Nephrol. 2020;21(1):50. doi:10.1186/s12882-02001718-8

10. Lin M, Liu C, Liu Y, et al. Fetuin-B links nonalcoholic fatty liver disease to chronic kidney disease in obese Chinese adults: a Cross-Sectional Study. Ann Nutr Metab. 2019;74(4):287-295. doi:10. 1159/000499843

11. Lee YJ, Wang CP, Hung WC, et al. Common and unique factors and the bidirectional relationship between chronic kidney disease and nonalcoholic fatty liver in type 2 diabetes patients. Diabetes Metab Syndr Obes. 2020;13:1203-1214. doi:10.2147/DMSO.S237700

12. Sirota JC, McFann K, Targher G, Chonchol M, Jalal DI. Association between nonalcoholic liver disease and chronic kidney disease: an ultrasound analysis from NHANES 1988-1994. Am J Nephrol. 2012;36(5):466-471. doi:10.1159/000343885

13. Akahane T, Akahane M, Namisaki T, et al. Association between non-alcoholic fatty liver disease and chronic kidney disease: a Cross-Sectional Study. J Clin Med. 2020;9(6):1635. doi:10.3390/ jcm9061635

14. Musso G, Gambino R, Tabibian JH, et al. Association of non-alcoholic fatty liver disease with chronic kidney disease: a systematic review and meta-analysis. PLoS Med. 2014;11(7): e1001680. doi:10.1371/journal.pmed.1001680

15. Mantovani A, Zaza G, Byrne CD, et al. Nonalcoholic fatty liver disease increases risk of incident chronic kidney disease: a systematic review and meta-analysis. Metabolism. 2018;79:64-76. doi:10.1016/j.metabol.2017.11.003

16. Lin S, Huang J, Wang M, et al. Comparison of MAFLD and NAFLD diagnostic criteria in real world. Liver Int. 2020;40(9):2082-2089. doi:10.1111/liv.14548

17. Sun DQ, Jin Y, Wang TY, et al. MAFLD and risk of CKD. Metabolism. 2021;115:154433. doi:10.1016/j.metabol.2020.154433

18. Levey AS, Stevens LA, Schmid CH, et al. A new equation to estimate glomerular filtration rate. Ann Intern Med. 2009;150 (9):604-612. doi:10.7326/0003-4819-150-9-200905050-00006

19. Yilmaz Y, Byrne CD, Musso G. A single-letter change in an acronym: signals, reasons, promises, challenges, and steps ahead for moving from NAFLD to MAFLD. Expert Rev Gastroenterol Hepatol. 2020;1-8. doi:10.1080/17474124.2021.1860019

20. Gheith O, Farouk N, Nampoory N, Halim MA, Al-Otaibi T. Diabetic kidney disease: world wide difference of prevalence and risk factors. J Nephropharmacol. 2016;5(1):49-56.

21. Fioretto P. Diabetic kidney disease: the onset of a new era? J Nephrol. 2020;33(5):899-900. doi:10.1007/s40620-020-00849-3
22. Russo GT, De Cosmo S, Viazzi F, et al. Diabetic kidney disease in the elderly: prevalence and clinical correlates. BMC Geriatr. 2018;18 (1):38. doi:10.1186/s12877-018-0732-4

23. Sharaf El Din UAA, Salem MM, Abdulazim DO. Uric acid in the pathogenesis of metabolic, renal, and cardiovascular diseases: a review. J Adv Res. 2017;8(5):537-548. doi:10.1016/j.jare.2016.11. 004

24. De Becker B, Borghi C, Burnier M, van de Borne P. Uric acid and hypertension: a focused review and practical recommendations. $J$ Hypertens. 2019;37(5):878-883. doi:10.1097/HJH.000000000000 1980

25. Oh TR, Choi HS, Kim CS, et al. Hyperuricemia has increased the risk of progression of chronic kidney disease: propensity score matching analysis from the KNOW-CKD study. Sci Rep. 2019;9(1):6681. doi:10.1038/s41598-019-43241-3

26. Bonino B, Leoncini G, Russo E, Pontremoli R, Viazzi F. Uric acid in CKD: has the jury come to the verdict? J Nephrol. 2020;33 (4):715-724. doi:10.1007/s40620-020-00702-7

27. Srivastava A, Kaze AD, McMullan CJ, Isakova T, Waikar SS. Uric Acid and the risks of kidney failure and death in individuals with CKD. Am J Kidney Dis. 2018;71(3):362-370.

28. Lora CM, Ricardo AC, Chen J, et al. Prevalence, awareness, and treatment of hypertension in hispanics/latinos with CKD in the Hispanic Community Health Study/Study of Latinos. Kidney Med. 2020;2(3):332-340. doi:10.1016/j.xkme.2020.02.005

29. Babu M, Drawz P. Masked hypertension in CKD: increased prevalence and risk for cardiovascular and renal events. Curr Cardiol Rep. 2019;21(7):58. doi:10.1007/s11886-019-1154-4

30. Gabriele MM, Koch Nogueira PC. Management of hypertension in CAKUT: protective factor for CKD. Front Pediatr. 2019;7:222. doi: $10.3389 /$ fped.2019.00222

31. Initiative CfDCaP-CKD. Chronic Kidney Disease in the United States, 2019; 2019.

32. Pan LL, Zhang HJ, Huang ZF, et al. Intrahepatic triglyceride content is independently associated with chronic kidney disease in obese adults: a cross-sectional study. Metabolism. 2015;64(9):1077-1085. doi:10.1016/j.metabol.2015.06.003

33. Chalasani N, Younossi Z, Lavine JE, et al. The diagnosis and management of nonalcoholic fatty liver disease: practice guidance from the American Association for the Study of Liver Diseases. Hepatology. 2018;67(1):328-357.

34. European Association for the Study of the L, European Association for the Study of D, European Association for the Study of O. EASLEASD-EASO clinical practice guidelines for the management of non-alcoholic fatty liver disease. J Hepatol. 2016;64(6):1388-1402. doi:10.1016/j.jhep.2015.11.004

35. Eslam M, Sarin SK, Wong VW, et al. The Asian Pacific Association for the Study of the Liver clinical practice guidelines for the diagnosis and management of metabolic associated fatty liver disease. Hepatol Int. 2020;14(6):889-919.

36. Eren F, Kaya E, Yilmaz Y. Accuracy of Fibrosis-4 index and non-alcoholic fatty liver disease fibrosis scores in metabolic (dysfunction) associated fatty liver disease according to body mass index: failure in the prediction of advanced fibrosis in lean and morbidly obese individuals. Eur J Gastroenterol Hepatol. 2020. doi:10.1097/ MEG.0000000000001946

37. McPherson S, Hardy T, Dufour J-F, et al. Age as a confounding factor for the accurate non-invasive diagnosis of advanced NAFLD fibrosis. Am J Gastroenterol. 2017;112(5):740-751. doi:10.1038/ ajg.2016.453 


\section{Publish your work in this journal}

Diabetes, Metabolic Syndrome and Obesity: Targets and Therapy is an international, peer-reviewed open-access journal committed to the rapid publication of the latest laboratory and clinical findings in the fields of diabetes, metabolic syndrome and obesity research. Original research, review, case reports, hypothesis formation, expert opinion and commentaries are all considered for publication. The manuscript management system is completely online and includes a very quick and fair peer-review system, which is all easy to use. Visit http://www.dovepress.com/testimonials.php to read real quotes from published authors.

Submit your manuscript here: https:/www.dovepress.com/diabetes-metabolic-syndrome-and-obesity-targets-and-therapy-journal 